doi:10.13108/2020-12-1-3

\title{
ALMOST PERIODIC AT INFINITY SOLUTIONS TO INTEGRO-DIFFERENTIAL EQUATIONS WITH NON-INVERTIBLE OPERATOR AT DERIVATIVE
}

\author{
M.S. BICHEGKUEV
}

\begin{abstract}
In the paper we consider an integro-differential equation with a non-invertible operator at a derivative in the space of uniformly continuous bounded functions. The integral part of the operator is a convolution of an operator-valued compactly supported Borel measure and a bounded continuous vector function. We obtain sufficient conditions (spectral conditions) of almost periodicity at infinity for bounded solutions of this equation.

The above results are based on the proven statement that if the right-hand side of the equation in question belongs to $C_{0}(\mathbb{J}, X)$, which is the space of functions tending to zero at infinity, then the Beurling spectrum of each weak solution is contained in the singular set of a characteristic equation. In particular, for the equations of the form $\mu * x=\psi$, where the function $\psi \in C_{0}(\mathbb{J}, X)$ and the support supp $\mu$ of a scalar measure $\mu$ are compact, we establish that each classical solution is almost periodic at infinity. We show that if the singular set of the characteristic function of the considered equation has no accumulation points in $\mathbb{R}$, then each weak solution is almost periodic at infinity. We study the structure of bounded solutions in terms of slowly varying at infinity functions.

We provide applications of our results to nonlinear integro-differential equations. We establish that when the right hand side of a nonlinear integro-differential equation is a decaying at infinity mapping and a singular set of the characteristic function has no finite accumulation points on $\mathbb{R}$, a bounded solution of this equation is almost periodic at infinity.

The main results of the paper are obtained by means of the methods of abstract harmonic analysis. The spectral theory of Banach modules is essentially employed.
\end{abstract}

Keywords: almost periodic at infinity function, Banach space of almost periodic functions at infinity, Beurling spectrum, Bohr almost periodic function.

Mathematics Subject Classification: 47G20

\section{INTRODUCTION AND MAIN RESULTS}

The theory of almost periodic functions created by H. Bohr [1] has numerous applications in studying the almost periodicity in the Bohr sense of bounded solutions to various classes of equations (differential, difference ones and others), see [2]-[14]. Usually one considered various classes of linear equations with almost periodic in the Bohr sense coefficients and the right hand side. However, a bounded solution to the simplest differential equation $\dot{x}(t)=A x(t)+\psi(t)$, $t \geqslant 0$, considered in a finite-dimensional linear space with a linear operator $A$ and a continuous vanishing at infinity function $\psi: \mathbb{R}_{+} \rightarrow \mathbb{R}$ is not almost periodic in the usual sense.

In papers by A.G. Baskakov [17, [18, a new class of continuous almost periodic functions was introduced; they are called almost periodic at infinity functions. This class involve almost

M.S. Bichegkuev, Almost Periodic an infinity solutions to integro-Differential EQUATIOns WITH NON-INVERTIBLE OPERATOR AT DERIVATIVE.

(C)Bichegkuev M.S. 2020.

Submitted April 30, 2019. 
periodic in the Bohr sense functions are well as the solutions of various classes of equations including the above discussed equation.

In the present work we obtain sufficient spectral conditions ensuring the almost periodicity at infinity for a rather wide class of integro-differential equations with a non-invertible equations at the derivative. The main results of the paper are contained in Theorems 1-4 and Theorem 7 . They are obtained by means of the methods in the abstract harmonic analysis. We also employ essentially the spectral theory of Banach moduli, see [11], [12], [19], [20], over the Banach algebra $L^{1}(\mathbb{R})=L^{1}(\mathbb{R}, \mathbb{C})$ of summable on $\mathbb{R}$ complex-valued functions with the convolution

$$
(f * g)(t)=\int_{\mathbb{R}} f(t-s) g(s) d s, \quad t \in \mathbb{R}, \quad f, g \in L^{1}(\mathbb{R})
$$

as the product.

We first introduce main functional space and several equivalent definitions of almost periodic at infinity functions.

Let $X$ be a complex Banach space and $L B(X)$ be a Banach algebra of linear bounded operators acting in $X$.

Let $\mathbb{J}$ be one of the segments $\mathbb{R}=(-\infty, \infty)$ or $\mathbb{R}_{+}=[0, \infty)$. By $C_{b}=C_{b}(\mathbb{J}, X)$ we denote the Banach space of continuous and bounded on $\mathbb{J}$ functions with values in $X$ and the norm

$$
\|x\|_{\infty}=\sup _{t \in \mathbb{J}}\|x(t)\|_{X} .
$$

The symbol $C_{b, u}=C_{b, u}(\mathbb{J}, X)$ stands for a closed subspace of uniformly continuous functions in $C_{b}(\mathbb{J}, X)$, while $C_{0}=C_{0}(\mathbb{J}, X)$ denotes a closed subspace of the functions in $C_{b}(\mathbb{J}, X)$ tending to zero at infinity.

In the Banach space $C_{b, u}(\mathbb{J}, X)$ we consider a strongly continuous semi-group of the operators $S: \mathbb{J} \rightarrow L B\left(C_{b, u}\right)$ acting by the rule:

$$
(S(t) x)(\tau)=x(t+\tau), t, \tau \in \mathbb{J}, x \in C_{b, u}(\mathbb{J}, X) .
$$

We note that $S$ is a group if $\mathbb{J}=\mathbb{R}$.

Definition 1. (see [17],[18]). The function $x \in C_{b, u}(\mathbb{J}, X)$ is called slowly varying at infinity if $(S(t) x-x) \in C_{0}(\mathbb{J}, X)$ for each $t \in \mathbb{J}$. We denote the set of slowly varying at infinity functions by the symbol $C_{s l, \infty}(\mathbb{J}, X)$.

The examples of slowly varying at infinity functions are

1) $x_{1}(t)=\sin \ln \left(1+t^{2}\right), t \in \mathbb{R}$;

2) $x_{2}(t)=\arctan t, t \in \mathbb{R}$;

3) $x_{3}: \mathbb{R}_{+} \rightarrow X, x_{3}(t)=c+x_{0}(t), t \geqslant 0$, where $c_{0}$ is a vector in the Banach space $X$ and $x_{0}$ is an arbitrary function from $C_{0}\left(\mathbb{R}_{+}, X\right)$;

4) each continuously differentiable function in $C_{b}(\mathbb{R}, X)$ obeying $\dot{x} \in C_{0}(\mathbb{R}, X)$.

We note that the set $C_{s l, \infty}(\mathbb{J}, X)$ is a closed subspace in the Banach space $C_{b, u}(\mathbb{J}, X)$.

In papers [17], [18], a definition of an almost periodic at infinity function was given. There are several approaches to defining such functions. The first definition is based on the notion of an $\varepsilon$-period, cf. [2.

Definition 2. Let $\varepsilon>0$. A number $\omega \in \mathbb{J}$ is called an $\varepsilon$-period at infinity of the function $x \in C_{b}(\mathbb{J}, X)$ if there exists a number $a(\varepsilon) \geqslant 0$ such that

$$
\sup _{|t| \geqslant a(\varepsilon)}\|x(t+\omega)-x(t)\|<\varepsilon .
$$

The set of $\varepsilon$-periods at infinity of the function $x$ is denoted by the symbol $\Omega_{\infty}(x ; \varepsilon)$. 
Definition 3. A function $x \in C_{b, u}(\mathbb{J}, X)$ is called almost periodic at infinity if for each $\varepsilon>0$ the set $\Omega_{\infty}(x ; \varepsilon)$ of its $\varepsilon$-periods possesses the following property: there exists a number $l(\varepsilon)>0$ such that each interval in $\mathbb{J}$ of the length $l(\varepsilon)$ contains at least one $\varepsilon$-period at infinity of the function $x$.

It follows from this definition corresponding to the Bohr definition of an almost periodic function [1] that each continuous almost periodic in the Bohr sense function $x \in C_{b}(\mathbb{J}, X)$ is almost periodic at infinity.

We are going to recall a series of definitions from the theorem of Banach moduli used in what follows. Let $\mathcal{X}$ be a complex Banach space. As a Banach algebra, we consider the space $L^{1}(\mathbb{R})=L^{1}(\mathbb{R}, \mathbb{C})$ with the convolution

$$
(f * g)(t)=\int_{\mathbb{R}} f(t-s) g(s) d s, \quad t \in \mathbb{R}, \quad f, g \in L^{1}(\mathbb{R}),
$$

as a product.

If $T: \mathbb{R} \rightarrow L B(\mathcal{X})$ is a strongly continuous isometric representation of the group $\mathbb{R}$, then the formula [19], [20]

$$
f x=\int_{\mathbb{R}} f(s) T(-s) x d s, \quad f \in L^{1}(\mathbb{R}), \quad x \in \mathcal{X},
$$

equips the space $\mathcal{X}$ by the structure of a Banach $L^{1}(\mathbb{R})$-module, which is also denoted by the symbol $(\mathcal{X}, T)$.

In particular, the Banach space $C_{b, u}(\mathbb{R}, \mathcal{X})=C_{b, u}$ is equipped by the structure of the Banach $L^{1}(\mathbb{R})$-module by means of the group of isometries of the translations of the functions

$$
S: \mathbb{R} \rightarrow L B\left(C_{b, u}\right),(S(t) x)(s)=x(s+t), \quad t, s \in \mathbb{R}, \quad x \in C_{b, u} .
$$

Thus, a module structure on $C_{b, u}$ is defined by the convolution of the functions

$$
(f * x)(t)=\int_{\mathbb{R}} f(t-s) x(s) x d s=\int_{\mathbb{R}} f(\tau)(S(-\tau) x)(t) d \tau, \quad t \in \mathbb{R},
$$

for all $f \in L^{1}(\mathbb{R}), x \in C_{b, u}$.

Definition 4. A vector $x$ in the Banach $L^{1}(\mathbb{R})$-module $(\mathcal{X}, T)$ is called almost periodic if the set of the vectors $\{T(t) x: t \in \mathbb{R}\}$, the orbit of the vector $x$, is pre-compact in $\mathcal{X}$.

The set of almost periodic vector in the Banach $L^{1}(\mathbb{R})$-module $\mathcal{X}$ forms a closed submodule denoted in what follows by the symbol $A P \mathcal{X}$. In particular, $A P C_{b, u}(\mathbb{R}, X)=A P(\mathbb{R}, X)$ is a Banach space of continuous almost periodic Bohr functions with respect to the group of translations $S(t), t \in \mathbb{R}$.

The symbol $\mathcal{X}$ stands for the quotient space $C_{b, u}\left(\mathbb{R}_{+}, X\right) / C_{0}\left(\mathbb{R}_{+}, X\right)$.

In the Banach space $\mathcal{X}$, the group of isometries $\tilde{S}: \mathbb{R} \rightarrow L B(\mathcal{X})$ acting by the rule

$$
\tilde{S}(t) \tilde{x}=\widetilde{S(t) x}, \quad t \in \mathbb{R}, \quad \tilde{x} \in \mathcal{X}
$$

is well-defined, where $S(t) x$ is the translation of the function $x$ to the left, see $(1)$, for $t \geqslant 0$, while as $t<0$, the symbol $\widetilde{S(t) x}$ denotes an equivalence class containing a continuous function of the form

$$
x_{t}(s)=\left\{\begin{aligned}
x(s+t), & \text { as } \quad s+t \geqslant 0, \\
-t^{-1} s x(0), & \text { as } \quad s+t \leqslant 0, \quad s \geqslant 0 .
\end{aligned}\right.
$$


The structure of Banach $L^{1}(\mathbb{R})$-module on $\mathcal{X}$ is introduce by the representation $\tilde{S}$, that is, by the formula

$$
f \tilde{x}=\int_{\mathbb{R}} f(\tau) \tilde{S}(-\tau) \tilde{x} d \tau, \quad f \in L^{1}(\mathbb{R}), \quad \tilde{x} \in \mathcal{X} .
$$

Remark 1. The definition of the module structure on the quotient space $\mathcal{X}=$ $C_{b, u}\left(\mathbb{R}_{+}, X\right) / C_{0}\left(\mathbb{R}_{+}, X\right)$ implies immediately that for all functions $f \in L^{1}(\mathbb{R})$ and $x \in$ $C_{b, u}\left(\mathbb{R}_{+}, X\right)$ the identity

$$
\widetilde{f x}=\left(\widetilde{f * y)\left.\right|_{\mathbb{R}_{+}}}\right.
$$

holds true for each continuation $y \in C_{b, u}(\mathbb{R}, X)$ of the function $x$ on $\mathbb{R}$ with the property:

$$
\lim _{t \rightarrow-\infty} y(t)=0 \text {. }
$$

Definition 5. A function $x \in C_{b, u}(\mathbb{J}, X)$ is called almost periodic at infinity if the equivalence class $\tilde{x}=x+C_{0}(\mathbb{J}, X)$ is an almost periodic vector in the space $\mathcal{X}=C_{b, u}(\mathbb{J}, X) / C_{0}(\mathbb{J}, X)$ of the ismetric representation $\tilde{S}: \mathbb{R} \rightarrow L B(\mathcal{X})$, that $i s,\{\tilde{S}(t) \tilde{x}: t \in \mathbb{R}\}$ is a pre-compact space in the quotient space $\mathcal{X}$ or, equivalently, $t \mapsto \tilde{S}(t) \tilde{x}: \mathbb{R} \rightarrow \mathcal{X}$ is a continuous almost periodic function.

Definition 6. A function $x \in C_{b, u}(\mathbb{J}, X)$ is called almost periodic at infinity if for each $\varepsilon>0$ there exist numbers $\lambda_{1}, \ldots, \lambda_{n} \in \mathbb{R}$ and slowly varying at infinity functions $x_{1}, \ldots, x_{n} \in$ $C_{s l, \infty}(\mathbb{J}, X)$ such that

$$
\sup _{t \in \mathbb{J}}\left\|x(t)-\sum_{k=1}^{n} x_{k}(t) e^{i \lambda_{k} t}\right\|<\varepsilon .
$$

The formulations of Definitions 5 and 6 imply that they are equivalent, see [17], [18].

The symbol $A P(\mathbb{I}, X)$ denotes a Banach space of almost periodic functions, while the symbol $A P_{\infty}(\mathbb{J}, X)$ stands for the Banach space of almost periodic at infinity functions in $C_{b, u}(\mathbb{J}, X)$.

It is clear that $C_{0}(\mathbb{J}, X) \subset C_{s l, \infty}(\mathbb{J}, X) \subset A P_{\infty}(\mathbb{J}, X)$.

By $\widehat{f}: \mathbb{R} \rightarrow \mathbb{C}$ we denote the Fourier transform

$$
\widehat{f}(\lambda)=\int_{\mathbb{R}} f(t) e^{-i \lambda t} d t, \quad \lambda \in \mathbb{R}
$$

of a function $f \in L^{1}(\mathbb{R})$.

Definition 7. The Beurling spectrum of a vector $x$ in a Banach $L^{1}(\mathbb{R})$-module $(\mathcal{X}, \widetilde{S})$ is the set

$$
\Lambda(x)=\left\{\lambda_{0} \in \mathbb{R}: \text { fx } \neq 0 \text { for each function } f \in L^{1}(\mathbb{R}) \text { obeying } \widehat{f}\left(\lambda_{0}\right) \neq 0\right\} .
$$

This definition implies that

$$
\Lambda(x)=\mathbb{R} \backslash\left\{\mu_{0} \in \mathbb{R}: \exists f \in L^{1}(\mathbb{R}) \text { such that } \widehat{f}\left(\mu_{0}\right) \neq 0 \text { and } f x=0\right\} .
$$

If $\mathcal{X}_{0}$ is a closed submodule in $\mathcal{X}$, then the quotient space $\mathcal{X} / \mathcal{X}_{0}$ is a Banach $L^{1}(\mathbb{R})$-module with a structure defined for all $f \in L^{1}(\mathbb{R})$ and $\tilde{x}=x+\mathcal{X}_{0}$ by the formula

$$
f \tilde{x}=f x+\mathcal{X}_{0}=\widetilde{f x} .
$$

Definition 8. Let $x \in C_{b, u}(\mathbb{J}, X)$. The Beurling spectrum of the function $x$ at infinity is the Beurling spectrum $\Lambda(\widetilde{x})$, where $\widetilde{x}=x+C_{0}(\mathbb{J}, X)$ is the equivalence class in $\mathcal{X}=$ $C_{b, u}(\mathbb{J}, X) / C_{0}(\mathbb{J}, X)$ and it is denoted by the symbol $\Lambda_{\infty}(x)$. 
We consider an integro-differential equation of the form

$$
B \dot{x}(t)+A x(t)+(\mu * x)(t)=\psi(t), t \in \mathbb{J},
$$

where $B, A \in L B(X, Y)$ are operators, $\mu: \sigma \rightarrow L B(X, Y)$ is the Borel measure with a compact support and $\sigma$ is the algebra of Borel sets in $\mathbb{J}$, and $\psi \in C_{b, u}(\mathbb{J}, Y)$. Hereinafter the symbol $L B(X, Y)$ stands for the Banach space of linear bounded operators acting from the Banach space $X$ with the values in $Y$. The operator $B$ is not necessarily invertible. The convolution $\mu * x$ of the measure $\mu$ and the function $x \in C_{b}(\mathbb{J}, Y)$ is defined by the formula

$$
(\mu * x)(t)=\int_{\mathbb{J}} \mu(d s) x(t-s), \quad t \in \mathbb{J} .
$$

Such form of the convolution is because the measure $\mu$ is operator-valued.

Definition 9. An operator valued function

$$
H: \mathbb{R} \rightarrow L B(X, Y), H(\lambda)=i B \lambda+A+\widehat{\mu}(\lambda), \lambda \in \mathbb{R},
$$

where $\widehat{\mu}(\lambda)=\int_{\mathbb{R}} \mu(d t) e^{-i \lambda t}, \lambda \in \mathbb{R}$, is the Fourier transform of the measure $\mu$, is called the characteristic function corresponding to operator equation (2).

Remark 2. Since the support $\operatorname{supp} \mu$ of the measure $\mu$ is compact, that is, the support $\operatorname{supp} \mu$ is contained in some segment $[a, b]$, it follows from the representation

$$
\widehat{\mu}(\lambda)=\int_{\mathbb{R}} \mu(d t) e^{-i \lambda t}=\int_{a}^{b} \mu(d t) e^{-i \lambda t}, \quad \lambda \in \mathbb{R},
$$

that the function $\widehat{\mu}: \mathbb{R} \rightarrow L B(X, Y)$ is infinitely differentiable on $\mathbb{R}$. Moreover, it can be continued to an entire function on $\mathbb{C}$ of exponential type at most $\max \{|b|,|a|\}$.

Definition 10. The set

$$
s(H)=\{\lambda \in \mathbb{R}: H(\lambda) \quad \text { is an invertible operator in } L B(X, Y)\}
$$

is called a singular set of the characteristic function $H$. The set $\rho(H)=\mathbb{R} \backslash s(H)$ is called a regular set of the function $H$.

Remark 3. Let $t_{k}, k \geqslant 1$, be some sequence of the points in the segment $[a, b]$ and $A_{k}$, $k \geqslant 1$, be a sequence of the operators in the Banach space $L B(X, Y)$ satisfying the condition $\sum_{k \geqslant 1}\left\|A_{k}\right\|<\infty$. Then the measure $\mu=\sum_{k \geqslant 1} A_{k} \delta_{t_{k}}$, where $\delta_{k}$ is the Dirac measure concentrated at a point $t_{k}$ satisfies the above conditions. Its Fourier transform reads as $\widehat{\mu}(\lambda)=\sum_{k \geqslant 1} A_{k} e^{-i \lambda t_{k}}$. Thus, $\mu$ is an almost periodic operator-valued function. In this case the characteristic function $H(\lambda)$ corresponding to equation (2) with the above measure $\mu$ reads as

$$
H(\lambda)=i B \lambda+A+\sum_{k \geqslant 1} A_{k} e^{-i \lambda t_{k}}, \quad \lambda \in \mathbb{R} .
$$

If $B=0$, then equation (2) becomes a difference equation of form

$$
A x(t)+\sum_{k \geqslant 1} A_{k} x\left(t-t_{k}\right)=\psi(t), \quad t \in \mathbb{J} .
$$


Remark 4. The considered class of equations includes a series of partial differential equations, where $A: D(A) \subset Y \rightarrow Y$ is a linear closed operator and $B$ is an operator relatively bounded with respect to the operator $A$. In this case $X=D(A)$ with the norm of the graph of the operator $A$. If $\mu=0$, then equation (2) becomes

$$
B \dot{x}(t)+A x(t)=\psi(t), \quad t \in \mathbb{J} .
$$

A characteristic function corresponding to this equation is the operator pencil

$$
H(\lambda)=i \lambda B+A, \quad \lambda \in \mathbb{R} .
$$

$A$ singular set of such characteristic function reads as

$$
s(H)=\{\lambda \in \mathbb{R}: i \lambda B+A \text { is an invertible operator }\} .
$$

Employing the terminology of [10]-[12], we obtain that the set $s(H)$ coincides with the set $\sigma(A,-B) \cap(i \mathbb{R})$, where $\sigma(A,-B)$ is the spectrum of an ordered pair $A,-B$, which is the spectrum of the operator pencil $A+\lambda B, \lambda \in \mathbb{R}$.

Many of the obtained results can be generalized to differential inclusions defined by linear relations on a Banach space, see [21]-[24].

Remark 5. The convolution equation of the form $\mu * x=\psi$ (the case $B=0, A=0$ ) was considered in paper [10]. In this paper there were obtained sufficient conditions of an asymptotic almost periodicity of this solution to this equation with a complex measure. We recall that the function $x \in C_{b, u}\left(\mathbb{R}_{+}, X\right)$ is called asymptotically almost periodic if it can be represented as a sum of two functions, one being almost periodic in the Bohr sense, while the other belongs to the space $C_{0}\left(\mathbb{R}_{+}, X\right)$. It is clear that such functions are almost periodic at infinity. Criterions for asymptotic almost periodicity of bounded solutions to parabolic equations were considered in papers [13]-[15], see also monograph [3].

Definition 11. A classical solution to integro-differential equation (2) is a continuously differentiable function $x_{0} \in C_{b, u}(\mathbb{J}, X)$ such that $\dot{x}_{0} \in C_{b, u}(\mathbb{J}, X)$ and it solves equation (2). A function $y_{0} \in C_{b, u}(\mathbb{J}, X)$ is called a weak solution to integro-differential equation (2) if it is a uniform limit of some sequence of classical solutions to equation (2).

The following four theorems present main results of the paper.

Theorem 1. For each function $\psi \in C_{0}(\mathbb{J}, X)$ and for each weak solution $x_{0}$ of equation (2) the inclusion holds:

$$
\Lambda_{\infty}\left(x_{0}\right) \subset s(H)
$$

This theorem serves as a base of most statements in this paper.

Theorem 2. Let the singular set $s(H)$ of a characteristic function $H$ have no finite accumulation points on $\mathbb{R}$ and let $\psi \in C_{0}(\mathbb{J}, X)$. Then each weak solution $x_{0} \in C_{b, u}(\mathbb{J}, X)$ is an almost periodic at infinity function.

Theorem 2 implies immediately the following statement.

Theorem 3. Let the singular set $s(H)$ of a characteristic function $H$ have no finite accumulation points on $\mathbb{R}$. Then each weak solution $x_{0} \in C_{b, u}(\mathbb{J}, X)$ of homogeneous equation (2) is an almost periodic at infinity function.

Theorem 4 (On the structure of bounded solutions). Let the set $s(H)=\left\{\lambda_{k}: k \geqslant 1\right\}$ have no finite accumulation points on $\mathbb{R}$ and $x_{0} \in C_{b, u}(\mathbb{J}, X)$ is a weak solution to equation (2) with 
the function $\psi \in C_{0}(\mathbb{J}, X)$. Then for each $\varepsilon>0$ there exist slowly varying at infinity functions $\varphi_{1}, \ldots, \varphi_{n} \in C_{s l, \infty}(\mathbb{J}, X)$ such that

$$
\sup _{t \in \mathbb{J}}\left\|x_{0}(t)-\sum_{k=1}^{n} \varphi_{k}(t) e^{i \lambda_{k} t}\right\|<\varepsilon .
$$

\section{PROOF OF MAIN RESUlTS}

We begin with the following lemma.

Lemma 1. A function $x_{0} \in C_{b, u}\left(\mathbb{R}_{+}, X\right)$ is almost periodic at infinity if and only if when the function the same holds for a function $y_{0} \in C_{b, u}(\mathbb{R}, X)$ with the following properties:

1) $y_{0}$ is a continuous continuation of $x_{0}$ on $\mathbb{R}$;

2) $\operatorname{supp} y_{0} \cap \mathbb{R}_{-}$is a compact set, where $\mathbb{R}_{-}=(-\infty, 0]$.

Proof. For an arbitrary $\omega>0$ there exist numbers $a(\omega)>0$ and $b(\omega)>0$ such that the identity holds:

$$
\sup _{t \geqslant a(\omega)}\left\|x_{0}(t+\omega)-x_{0}(t)\right\|=\sup _{t \notin[-b(\omega), a(\omega)]}\left\|y_{0}(t \pm \omega)-y_{0}(t)\right\| .
$$

This identity implies immediately that

$$
\begin{aligned}
& \Omega_{\infty}\left(x_{0}, \varepsilon\right)=\Omega_{\infty}\left(y_{0}, \varepsilon\right) \cap \mathbb{R}_{+}, \\
& \Omega_{\infty}\left(y_{0}, \varepsilon\right)=\Omega_{\infty}\left(x_{0}, \varepsilon\right) \cup\left(-\Omega\left(x_{0}, \varepsilon\right)\right),
\end{aligned}
$$

for each $\varepsilon>0$.

Proof of Theorem 1. We first assume that $x_{0}$ is a classical solution to equation (2). Let $\lambda_{0} \in$ $\rho(H)=\mathbb{R} \backslash s(H)$. The function $H(\lambda)=i \lambda B+A+\hat{\mu}(\lambda), \lambda \in \mathbb{C}$, is entire. This is why the invertibility of $H\left(\lambda_{0}\right)$ yields the existence of a number $\delta>0$ such that the interval $\left(\lambda_{0}-\delta, \lambda_{0}+\delta\right)$ is contained in a regular set $\rho(H)$ of the function $H$; the number $\delta$ is determined by the condition

$$
\sup _{\left|\lambda-\lambda_{0}\right|<\delta}\left\|H(\lambda)-H\left(\lambda_{0}\right)\right\| \cdot\left\|H\left(\lambda_{0}\right)\right\|^{-1}<1 .
$$

Thus, $H(\lambda), \lambda \in\left(\lambda_{0}-\delta, \lambda_{0}+\delta\right)$, are invertible operators.

Let us consider an infinitely differentiable function $f_{0}$ in the algebra $L^{1}(\mathbb{R})$ with the properties:

1) $\widehat{f}_{0}\left(\lambda_{0}\right) \neq 0$

2) $\operatorname{supp} \widehat{f}_{0} \subset\left(\lambda_{0}-\delta, \lambda_{0}+\delta\right)$.

Then the function

$$
\widehat{F}(\lambda)=\left\{\begin{array}{ccc}
\widehat{f}_{0}(\lambda) H(\lambda)^{-1}, & \text { as } & \lambda \in\left(\lambda_{0}-\delta, \lambda_{0}+\delta\right), \\
0, & \text { as } & \lambda \notin\left(\lambda_{0}-\delta, \lambda_{0}+\delta\right),
\end{array}\right.
$$

is infinitely differentiable and is compactly supported. It is a Fourier transform of a summable operator-valued function $F: \mathbb{R} \rightarrow L B(Y, X)$ of the form

$$
F(t)=\frac{1}{2 \pi} \int_{\mathbb{R}} \widehat{F}(\lambda) e^{i \lambda t} d \lambda, \quad t \in \mathbb{R} .
$$

We first consider the case $\mathbb{J}=\mathbb{R}$. We apply the operator of the convolution with the function $F$ with the identity

$$
B \dot{x}_{0}+A x_{0}+\mu * x_{0}=\psi .
$$

As a result, we get the identity by employing the simplest properties of the Fourier transform:

$$
(B \dot{F}+A F+\mu * F) * x_{0}=F * \psi .
$$


Hence, the relation holds:

$$
\Phi * x_{0}=F * \psi=\varphi \in C_{0}(\mathbb{R}, X),
$$

where $\Phi: \mathbb{R} \rightarrow L B(Y, X)$ is a summable operator-valued function of the form

$$
\Phi=B \dot{F}+A F+\mu * F .
$$

Its Fourier transform reads as

$$
\begin{aligned}
\widehat{\Phi}(\lambda) & =i \lambda B \widehat{F}(\lambda)+A \widehat{F}(\lambda)+\widehat{\mu}(\lambda) \widehat{F}(\lambda) \\
& =\left\{\begin{array}{cl}
H(\lambda) \widehat{F}(\lambda), & \text { as } \quad \lambda \in\left(\lambda_{0}-\delta, \lambda_{0}+\delta\right), \\
0, & \text { as } \quad \lambda \notin\left(\lambda_{0}-\delta, \lambda_{0}+\delta\right),
\end{array} \quad \lambda \in \mathbb{R} .\right.
\end{aligned}
$$

Since $\widehat{f}_{0}(\lambda) I=H(\lambda) \widehat{F}(\lambda)$, where $\lambda \in\left(\lambda_{0}-\delta, \lambda_{0}+\delta\right)$ and $I$ is the identity mapping in $L B(Y)$, the identity holds:

$$
f_{0} * x_{0}=\varphi \in C_{0}(\mathbb{R}, X) .
$$

Since $\widehat{f}_{0}\left(\lambda_{0}\right) \neq 0$, in view of the definition of the Beurling spectrum at infinity we obtain that $\lambda_{0} \in \Lambda_{\infty}\left(x_{0}\right)$. This proves inclusion (3).

Let $\mathbb{J}=\mathbb{R}_{+}$and $x_{0} \in C_{b, u}\left(\mathbb{R}_{+}, X\right)$ is a solution to equation (2). We define a function $y_{0} \in C_{b, u}(\mathbb{R}, X)$ as in the lemma. Then the identity holds:

$$
B \dot{y}_{0}(t)+A y_{0}(t)+\left(\mu * y_{0}\right)(t)=\psi_{1}(t), \quad t \in \mathbb{R},
$$

where $\psi_{1} \in C_{b, u}(\mathbb{R}, Y), \psi_{1}(t)=\psi(t)$ as $t \geqslant \max \{0, b\}$. The function $\psi_{1}: \mathbb{R} \rightarrow Y$ is compactly supported on the semi-axis $(-\infty, b]$ due to the compactness of the support of the measure $\mu$. Hence, by Lemma 1 , the function $\psi_{1}$ belongs to the space $A P_{\infty}(\mathbb{R}, X)$. Remark 1 implies also the identity $\Lambda_{\infty}\left(y_{0}\right)=\Lambda_{\infty}\left(x_{0}\right)$. Therefore, $\Lambda_{\infty}\left(x_{0}\right) \subset s(H)$.

Let $x_{0}$ be a weak solution to equation $(2)$ and let $\left(x_{n}\right)$ be a sequence of classical solutions converging uniformly to $x_{0}$. By the above proven facts, $x_{n} \in A P_{\infty}(\mathbb{J}, X), n \in \mathbb{Z}$, and therefore, by the closedness of $A P_{\infty}(\mathbb{J}, X)$, we have $x_{0} \in A P_{\infty}(\mathbb{J}, X)$.

In what follows we make use of the following theorem.

Theorem 5. Assume that the Beurling spectrum at infinity $\Lambda_{\infty}\left(x_{0}\right)$ of the function $x_{0} \in$ $C_{b, u}(\mathbb{J}, X)$ has no accumulation points in $\mathbb{R}$. Then $x_{0} \in A P_{\infty}(\mathbb{J}, X)$.

A more abstract version of this theorem was provided in paper [11].

Proof of Theorem 2. Theorem 1 implies the inclusion $\Lambda_{\infty}\left(x_{0}\right) \subset s(H)$. Since the set $s(H)$ possesses no accumulation points on $\mathbb{R}$, according Theorem 5 , we have $x \in A P_{\infty}(\mathbb{J}, X)$.

Proof of Theorem 4. It follows from Theorems 1 and 2 that $x_{0} \in A P_{\infty}(\mathbb{J}, X)$ and $\Lambda\left(\widetilde{x}_{0}\right) \subset$ $s(H)=\left\{\lambda_{k}: k \geqslant 1\right\}$. The results of papers [17] and [18] yield that the Fourier series an almost periodic at infinity reads as $x(t)=\sum_{k \geqslant 1} \psi_{k}(t) e^{i \lambda_{k} t}$, where $\psi_{k}, k \geqslant 1$, are slowly varying at infinity functions. The theorem on approximation of an almost periodic at infinity function [18] implies that for each $\varepsilon>0$ there exist functions $\varphi_{1}, \ldots, \varphi_{n} \in C_{s l, \infty}(\mathbb{J}, X)$ such that inequality (3) holds true.

We consider the equation

$$
\mu * x=\psi,
$$

where the function $\psi \in C_{0}(\mathbb{J}, X)$ and the support supp $\mu$ of the scalar measure $\mu$ is compact. In this case, the characteristic function of this equation reads as

$$
H(\lambda)=\widehat{\mu}(\lambda), \quad \lambda \in \mathbb{R} .
$$


This is why $s(H)=\operatorname{supp} \widehat{\mu}$. Since $\widehat{\mu}$ can be continued to an entire function of an exponential type on the complex plane, according the uniqueness theorem for analytic functions, the set $s(H)$ can have no finite accumulation points on $\mathbb{R}$. Thus, we have just proved the following theorem.

Theorem 6. Each classical solution $x_{0} \in C_{b, u}(\mathbb{J}, X)$ to equation (4) is an almost periodic at infinity function.

\section{Almost PERIODIC AT INFINITY SOLUTIONS TO NONLINEAR EQUATIONS}

Definition 12. A continuous mapping $\varphi: \mathbb{J} \times X \rightarrow \mathbb{Y}$ is called decaying at infinity if

$$
\lim _{|t| \rightarrow \infty} \sup _{\|x\| \leqslant R}\|\varphi(t, x)\|=0
$$

for each $R>0$.

Theorem 7. Let $x_{0} \in C_{b, u}(\mathbb{J}, Y)$ be a bounded solution of a nonlinear differential equation

$$
B \dot{x}(t)+A x(t)+(\mu * x)(t)=\varphi(t, x),
$$

where $\varphi$ is a decaying at infinity mapping and let the set $s(H)$ have no finite accumulation point in $\mathbb{R}$. Then $x_{0}$ is an almost periodic at infinity function.

Proof. Since $x_{0}$ is a solution of equation (5), then $x_{0}$ solves also inhomogeneous linear integraldifferential equation (2), where $\psi(t)=\varphi\left(t, x_{0}(t)\right), t \in \mathbb{J}$. Definition 12 implies that the function $\psi$ belongs to the space $C_{0}(\mathbb{J}, Y)$. Thus, the function $x_{0}$ satisfies assumptions of Theorem 3 .

Theorem 7 implies the following statement.

Corollary 1. Let the assumptions of Theorem 7 be satisfied and the mapping $\varphi: \mathbb{J} \times X \rightarrow Y$ reads as $\varphi(t, x)=\varphi_{0}(t) g(x)$, where $g: X \rightarrow Y$ is a continuous mapping bounded on bounded sets and $\varphi \in C_{0}(\mathbb{J}, X)$. Then $x_{0}$ is an almost periodic at infinity function.

\section{BIBLIOGRAPHY}

1. H. Bohr. Almost periodic functions. OGIZ, Moscow (1934). [Dover Publications, Mineola (2018).]

2. B.M. Levitan, V.V. Zhikov. Almost periodic functions and differential equations. Izdat. MGU, Moscow (1978). [Cambridge Univ. Press, Cambridge (1982).]

3. W. Arendt, C.J.K. Batty. Vector-valued Laplace transforms and Cachy problems. Birkhäuser, Basel (2011).

4. B.M. Levitan. Integration of almost periodic functions with values in a Banach space // Izv. AN SSSR. Ser. Matem. 30:5, 1101-1110 (1966). (in Russian).

5. L. Amerio, G. Prouse. Almost-periodic functions and functional equations. Springer Verlag, New York (2013).

6. R. Doss. On almost periodic solutions of integro-differential// Ann. Math. 81:1, 117-123 (1965).

7. C. Foias, S. Zaidman. Almost-periodic solutions of parabolic sistem// Ann. Scuola Norm. Pisa. 3:3, $247-262(1963)$.

8. B.M. Lewitan. On a integral equation with almost periodic solutions // Bull.Amer. Math. Soc. 43:10, 677-679 (1937).

9. L.H. Loomis. The spectral characterization of a class of almost periodic functions // Ann. Math. 72:2, 362-368 (1960).

10. O. Staffans. On asymptotically almost periodic solutions of a convolution equation // Trans. Amer. Math. Soc. 226:2, 603-616 (1981).

11. A.G. Baskakov. Spectral criteria for almost periodicity of solutions of functional equations / Matem. Zamet. 24:2, 195-206 (1978). [Math. Notes. 24:2, 606-612 (1978).] 
12. A.G. Baskakov. Harmonic analysis of cosine and exponential operator-valued functions // Matem. Sborn. 124(166):1(5), 68-95 (1984). [Math. USSR-Sb. 52:1, 63-90 (1985).]

13. Yu.I. Lyubich, Q.Ph. V $\widetilde{u}$. Asymptotic stability of linear differential equations in Banach spaces // Studia Math. 88:1, 37-42 (1988).

14. W. Arendt, C.J.K. Batty. Tauberian theorems and stability of oneparameter semigroups // Trans. Amer. Math. 306:2, 837-852 (1988).

15. B. Basit. Harmonic analysis and asymptotic behavior of solutions to the abstract Cauchy problem // Semigroup Forum. 54:1, 58-74 (1997).

16. S. Bochner, J von Neuman. On compact solution of operational differential equations // Ann of Math. 36:1, 255-291 (1935).

17. A.G. Baskakov. Harmonic and spectral analysis of power bounded operators and bounded semigroups of operators on Banach spaces // Matem. Zamet. 98:2, 174-190 (2015). [Math. Notes. 98:2, 164-178 (2015).]

18. A.G. Baskakov. Analysis of linear differential equations by methods of the spectral theory of difference operators and linear relations // Uspekhi Matem. Nauk. 68:1(409), 77-128 (2013). [Russ. Math. Surv. 68:1, 69-116 (2013).]

19. A.G. Baskakov. Spectral synthesis in Banach modules over commutative Banach algebras // Matem. Zamet. 34:4, 776-782 (1983). [Math. Notes. 34:4, 776-782 (1983).]

20. A. G. Baskakov. Representation theory for Banach algebras, Abelian groups, and semigroups in the spectral analysis of linear operators // Sovrem. Matem. Fundam. Naprav. 9, 3-151 (2004). [J. Math. Sci. 137:4, 4885-5036 (2006).]

21. A.G. Baskakov, K.I. Chernyshov. Spectral analysis of linear relations and degenerate operator semigroups // Matem. Sborn. 193:11, 3-42 (2002). [Sb. Math. 193:11, 1573-1610 (2002).]

22. A.G. Baskakov. Linear relations as generators of semigroups of operators // Matem. Zamet. 84:2, 175-192 (2008). [Math. Notes. 84:2, 166-183 (2008).]

23. M.S. Bichegkuev. To the theory of infinitely differentiable semigroups of operators // Alg. Anal. 22:2, 1-13 (2010). [St. Petersburg Math. J. 22:2, 175-182 (2011).]

24. M.S. Bichegkuev. Lyapunov transformation of differential operators with unbounded operator coefficients // Matem. Zamet. 124:5, 68-95 (2016). [Math. Notes. 99:1, 24-36 (2016).]

Mairbek Suleimanovich Bichegkuev, North Ossetian State University named after K.L. Khetagurov, Vatutin str., 44-46, 362025, Vladikavkaz, Russia

E-mail: bichegkuev@yandex.ru 